\title{
Instabilité des versants et risques en montagne Perspectives pour une recherche régionale dans les Alpes du Nord
}

\author{
Jean-Pierre Asté*
}

Le domaine montagnard est un théâtre privilégié pour la manifestation des phénomènes d'instabilité des versants. Ils y revêtent leurs formes les plus variées dans des configurations morphologiques et géologiques très diversifiées, qui créent les conditions propices à leur déclenchement sous l'effet de facteurs aggravants d'origine tectonique, sismique, hydroclimatologique, ou tout simplement humaine.

Longtemps ignorée ou évitée, la montagne est aujourd'hui source d'intérêt et de richesse. Les phénomènes d'instabilité, par leur caractère essentiellement aléatoire, génèrent donc des risques pour les divers éléments du patrimoine montagnard.

\section{D'un programme national...}

Les partenaires réunis dans le cadre du groupe « Mouvements de terrain " du Contrat de Plan Etat-Région RhôneAlpes, outre le fait qu'ils développent une recherche à caractère régional dans les Alpes du Nord, amènent chacun une expérience spécifique de la prévention de ces risques :

- l'IRIGM, à titre universitaire, grâce à une bonne connaissance du milieu naturel et de son comportement, - l'ADRGT, association pour le développement de la recherche sur les glissements de terrain, sur la base de 20 années d'expérience,

- le CETE, grâce à son rôle privilégié au service du Ministère de l'Equipement,

- et le BRGM, par son expérience de la terre, au service des collectivités locales et des grands donneurs d'ordre.

(*) BRGM, coordinateur d'un groupe de travail réunissant : - MM. les Professeurs ANTOINE et GIRAud, de l'Université Joseph Fourier à St-Martin d'Hères.

- MM. Azimi et Desvarreux, de l'adrGT.

- M. Rotheval, du CETE de Lyon.
Dans le cadre du budget limité du premier programme Etat-Région (1986-1989), ces partenaires ont appris à se connaître et à identifier leurs forces et leurs faiblesses, puis à confronter leurs points de vue sur les divers aspects de la prévention des risques naturels en montagne. Le résultat de cette collaboration est exprimé sous forme d'un rapport en quatre chapitres (réf. 1) :

- Les mouvements de terrain - Mécanismes - Variété - Paramètres importants.

- Les mouvements de terrain du domaine alpin dans leur contexte géologique.

- Quelques aspects socio-économiques des mouvements de terrain.

- Méthodologie des actes de dépistage - Pronostic, surveillance et travaux de prévention.

Ce rapport fournit en outre une analyse de l'état des connaissances internationales sur le sujet, exprimée au symposium international de Lausanne en 1988.

Au terme de cette réflexion, deux voies semblaient envisageables :

- consolider, valider ou remettre en cause les connaissances et expériences acquises,

- poursuivre des recherches fondamentales sur la phénoménologie.

D'un commun accord, le groupe, dans un souci de réalisme et d'efficacité à court terme soutenu par le Conseil scientifique et le Comité de pilotage du Contrat, a choisi de privilégier la première voie, chacun des membres poursuivant cependant et par ailleurs des recherches plus fondamentales dans des domaines spécifiques. Une volonté de coordination de ces recherches fondamentales a cependant été exprimée. Elle sera confortée en 1991 par l'organisation par le Comité Français de Géologie de l'Ingénieur, à la demande conjointe de la Délégation aux Risques Majeurs du Ministère de l'Environnement et de la Direction de la 
Sécurité Civile du Ministère de l'Intérieur, d'un "conclave" sur les mouvements de terrain de grande ampleur, conclave qui portera sur les principaux thèmes de la connaissance des phénomènes et des situations associées à leur manifestation : géologie, mécanismes, surveillance et alerte, et enfin gestion des situations de crise.

Le programme de travail du Groupe pour la période 1990-1992 est donc basé sur les tâches suivantes:

- Création d'un Inventaire National des Versants Instables (INVI) (réf. 2).

Le prototype de cet inventaire a été développé par le BRGM avec le concours de la DRM et celui de la DSC (réf. 2), sous forme d'une base de données relationnelle intégrant des éléments d'information scientifiques, techniques, mais aussi économiques, administratifs, voire juridiques, sur les événements recensés. Sa version définitive permettra, dans le cadre du présent programme, de réunir toutes les informations (environ 3500 événements) disponibles sur les départements de l'Isère et de la Savoie. Le tableau de la figure 1 illustre partiellement un mode d'extraction de ces données sur une commune alpine.

- Mise au point d'une typologie régionale spécifique multicritères.

L'objectif assigné à cette tâche est de permettre de relier entre eux un certain nombre d'événements pouvant être considérés comme semblables sur des critères scientifiques et techniques ou sur des critères socio-économiques ou administratifs, afin de pouvoir procéder à des analyses consolidées de leurs manifestations et élargir, de ce fait, leur compréhension d'ensemble. Pour illustrer ce propos, on se réfèrera à la figure 2 , qui montre la répartition spatiale d'une série d'événements qui se sont produits simultanément pendant la période de janvier et février 1990, exceptionnelle par les conditions hydrométéorologiques qui ont régné sur les Alpes du Nord.

La figure 3, quant à elle, correspond à un essai de corrélation entre les grands ensembles lithologiques régionaux et les principaux types de mouvements pris en compte dans les classifications traditionnelles. Ce type de travail sera largement développé pendant le programme pour proposer de nouvelles approches plus pertinentes de l'identification des principaux types de phénomènes concernés ici.

- Analyse en retour d'un certain nombre de méthodes de prévention mises en auvre ces dernières années dans la région.

On a retenu essentiellement trois catégories principales de méthodes de prévention:

- La planification préventive.

Divers essais de cartographie des zones exposées à des mouvements de terrain ont été menés dans les Alpes depuis 20 ans. La détermination de l'extension des surfaces présumées instables relève en l'état actuel d'une forte subjectivité, dans la mesure où la variation spatiale de la plupart des facteurs qui permettent d'asseoir cette présomption est très aléatoire. Quant au terme d'occurrence dans le temps ou au rythme de réactivation des phénomènes concernés, il dépend de circonstances aggravantes inégalement contrôlables. L'objectif est donc d'affiner et d'harmoniser des analyses déjà établies sur quelques zones-test, puis d'essayer de proposer des méthodologies nouvelles basées sur les moyens modernes de traitement de l'information que sont les SGBD, les systèmes d'information géographique et autres outils d'analyse servis par des thématiciens de l'instabilité des pentes. La figure 4 montre, dans le cadre d'une étude pilote réalisée à Nantua, un extrait de carte ZERMOSS (1975) et un zonage plus récent d'exposition à des phénomènes de glissement ou de chutes de blocs rocheux (réf. 3).

- La surveillance des zones affectées.

Dans beaucoup de cas, la prévention a pour objectif essentiel de vivre avec un phénomène actif, trop important pour que l'on puisse espérer le juguler par des travaux. Dans ce cas, il faut exercer une surveillance constante du phénomène et de ses possibles interactions avec le patrimoine exposé. L'ambition, ici, est d'essayer de tirer un bilan de nombreuses expériences vécues dans la région, d'en montrer, pour les consolider, les aspects posịtifs, et d'établir des guides et recommandations pour résorber les difficultés ou carences que l'analyse aura mises en évidence.

- Les travaux de défense active ou passive.

Beaucoup de travaux coûteux ont été entrepris pour essayer de stabiliser des mouvements potentiels ou déclenchés. On ne bénéficie malheureusement que fort peu souvent d'analyses en retour sur l'efficacité réelle de ces travaux ou sur le coût et les conditions de leur maintenance. Il a donc semblé important de réaliser une enquête auprès des divers services maîtres d'ouvrage de tels travaux: services de l'Equipement, services de l'Agriculture, collectivités locales ou sociétés d'équipement en montagne.

Pour l'ensemble de ces tâches, il était important de bénéficier de l'information disponible dans ces services. En ce qui concerne ceux de l'Equipement, l'enquête sera menée auprès de quelques arrondissements ou subdivisions particulièrement concernés. Pour ce qui est de l'agriculture, compte tenu du rôle spécifique des services de Restauration des Terrains en Montagne, un protocole d'accord a été passé entre le groupe et la direction de ces services.

\section{...Vers un programme européen}

Par ailleurs, le groupe, l'Etat et la Région, déjà partenaires au plan national, ont décidé de porter le projet devant la Communauté Européenne dans le cadre de l'appel d'offres EPOCH (European Program on Climatology and Natural Hazards).

Pour cela, il a fallu trouver un partenaire étranger et une formulation plus large du projet.

Le partenaire choisi a été la Société Régionale pour le développement Agricole de la Lombardie (ERSAL), coordinatrice d'un groupe italien regroupant un certain nombre d'équipes de recherche de Florence, Milan et Venise. Ce groupement italien est spécialisé dans l'agriculture de montagne et la lutte contre la dégradation des terres par l'érosion. 


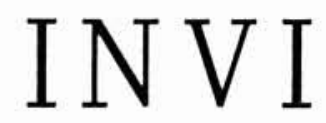

INVENTARE NATIONAL DES VERSANTS INSTABLES

1 - Mode de sortie imprimante des évènements relatifs à une commune

\begin{tabular}{|c|c|c|c|c|c|c|c|c|}
\hline N REF. & COMMUNE. & N INSEE & LOCALISATION & DATE & TYPE DE MOUVEIENT & ND MORTS & Nb BLES. & BIENS ENDONeMAGES \\
\hline $\begin{array}{ll}38 & 0774 \\
38 & 0775 \\
38 & 0776\end{array}$ & $\begin{array}{l}\text { MEAUDRE } \\
\text { MEAUDRE } \\
\text { MEAUDRE }\end{array}$ & $\begin{array}{l}225 \\
225 \\
225\end{array}$ & & $\begin{array}{l}\text { NON CONNUE } \\
\text { NON CONNUE } \\
\text { NON CONNUE }\end{array}$ & $\begin{array}{l}\text { GL ISSEMENT } \\
\text { CHUTE DE PIERRES } \\
\text { CRUE TORRENTIEL. }\end{array}$ & $\begin{array}{l}0 \\
0 \\
0\end{array}$ & $\begin{array}{l}0 \\
0 \\
0\end{array}$ & \\
\hline \begin{tabular}{|ll}
38 & 0575 \\
38 & 0574 \\
38 & 0573 \\
38 & 0778 \\
38 & 0777 \\
38 & 0075 \\
38 & 0055 \\
38 & 0960 \\
38 & 1055 \\
38 & 1056 \\
38 & 1058
\end{tabular} & $\begin{array}{l}\text { MENS } \\
\text { MENS } \\
\text { MENS } \\
\text { MENS } \\
\text { MENS } \\
\text { MENS } \\
\text { MENS } \\
\text { MENS } \\
\text { MENS } \\
\text { MENS } \\
\text { MENS }\end{array}$ & $\begin{array}{l}226 \\
226 \\
226 \\
226 \\
226 \\
226 \\
226 \\
226 \\
226 \\
226 \\
226\end{array}$ & $\begin{array}{l}\text { ROUTE DE CLELLES A MENS } \\
\text { TUNNEL DES ROCHERS } \\
\text { LES ROCHERS } \\
\\
\text { CD } 254 \text { MOULIN DE CHARDAYRE.AU } \\
\text { A CHARDAYRE, TOUS LES FONDS } \\
\text { CDS26 PK } 11,2 \text { a } 12,2 \\
\text { "LE FOREYRE" } \\
\text { RD DU RUISSEN DE MENS } \\
\text { "LE PONTILLARD" }\end{array}$ & $\begin{array}{l}\text { OCTOBRE } 1889 \\
16 / 05 / 1954 \\
28 / 02 / 1957 \\
\text { NON CONNUE } \\
\text { NON CONNUE } \\
\text { NON CONNUE } \\
\text { NON CONNUE } \\
\text { NON CONNUE } \\
\text { NON CONNUE } \\
\text { NON CONNUE } \\
\text { NON CONNUE }\end{array}$ & \begin{tabular}{|c|} 
ECROULEMENT \\
CHUTE DE BLOCS \\
ECROULEMENT \\
COULEE \\
CRUE TORRENTIEL. \\
GLISSEMENT \\
INCON. IINDETERM. \\
GLIS. DE TALUS \\
MV+ LENT DE VERS. \\
GLIS. DE TALUS \\
GLIS. DE TALUS
\end{tabular} & $\begin{array}{l}0 \\
0 \\
0 \\
0 \\
0 \\
0 \\
0 \\
0 \\
0 \\
0 \\
0\end{array}$ & $\begin{array}{l}0 \\
0 \\
0 \\
0 \\
0 \\
0 \\
0 \\
0 \\
0 \\
0 \\
0\end{array}$ & $\begin{array}{l}\text { ROUTE } \\
\text { ROUTE } \\
\text { ROUTE } \\
\text { ROUTE } \\
\text { CHAMP }\end{array}$ \\
\hline $\begin{array}{ll}38 & 0572 \\
38 & 0056\end{array}$ & $\begin{array}{l}\text { MEYLAN } \\
\text { MEYLAN }\end{array}$ & $\begin{array}{l}229 \\
229\end{array}$ & $\begin{array}{l}\text { VILLAGE DE LA BATIE } \\
\text { LA BATIE MEYLAN.ROCHER DE ST- }\end{array}$ & \begin{tabular}{|l|}
$18 / 12 / 1886$ \\
NON CONNUE
\end{tabular} & \begin{tabular}{|c|} 
ECROULEMENT \\
CHUTE DE PIERRES
\end{tabular} & $\begin{array}{l}0 \\
0\end{array}$ & $\begin{array}{l}0 \\
0\end{array}$ & \\
\hline
\end{tabular}

2 - Mode de sortie graphique

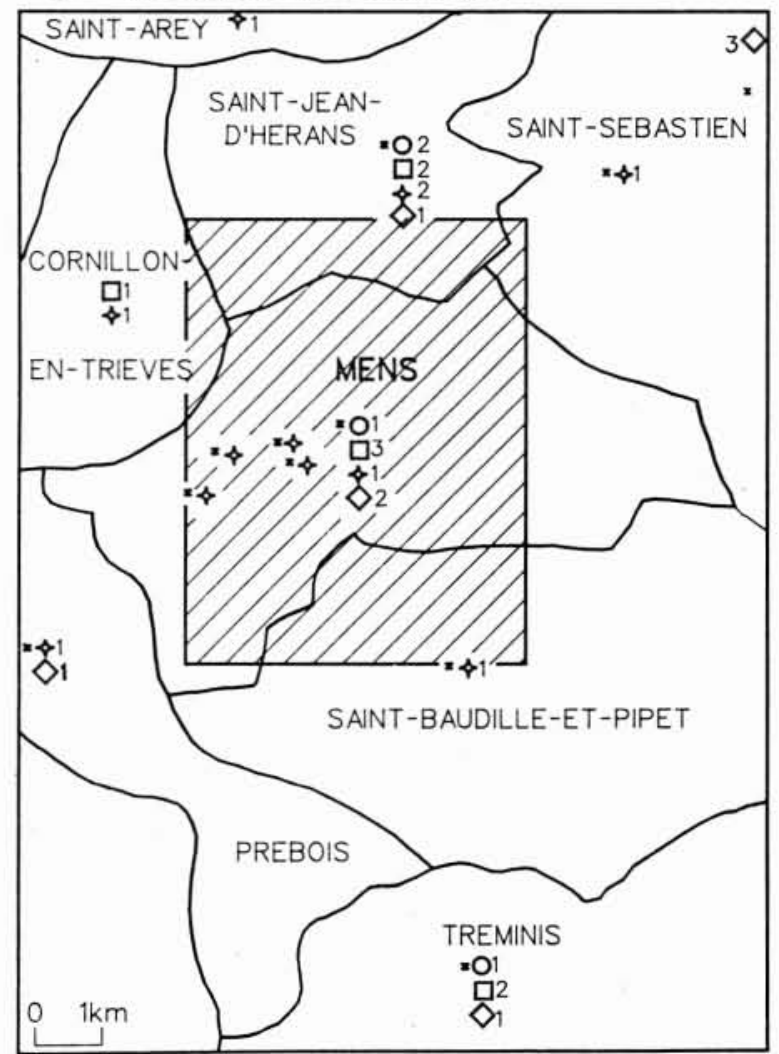

Localisation des évènements recensés

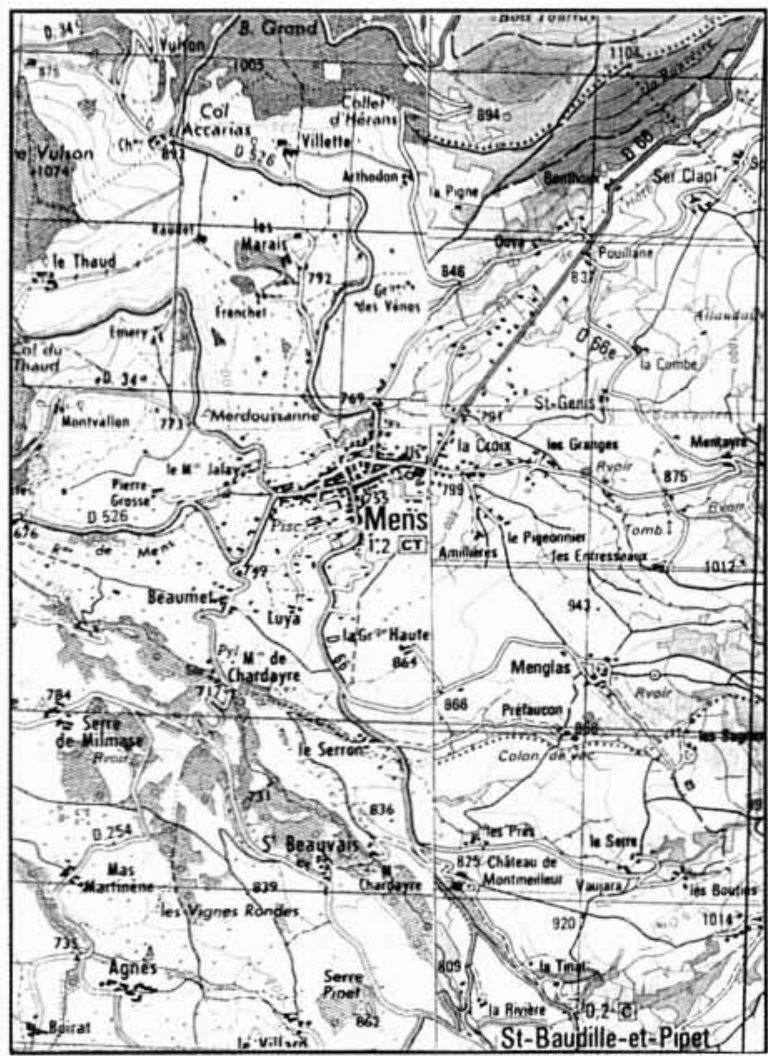

Extrait de la carte IGN 1/50 000

Commentaire : sur la commune de MENS, 11 évẻnements sont recensés.

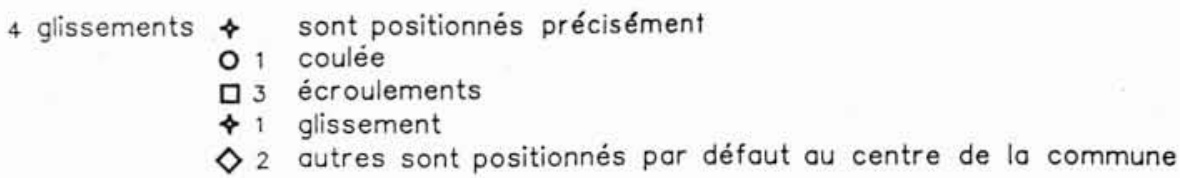

1. 
RECENSEMENT DES EVENEMENTS SURVENUS DANS LES ALPES DU NORD AUX MOIS DE JANVIER ET FEVRIER 1990

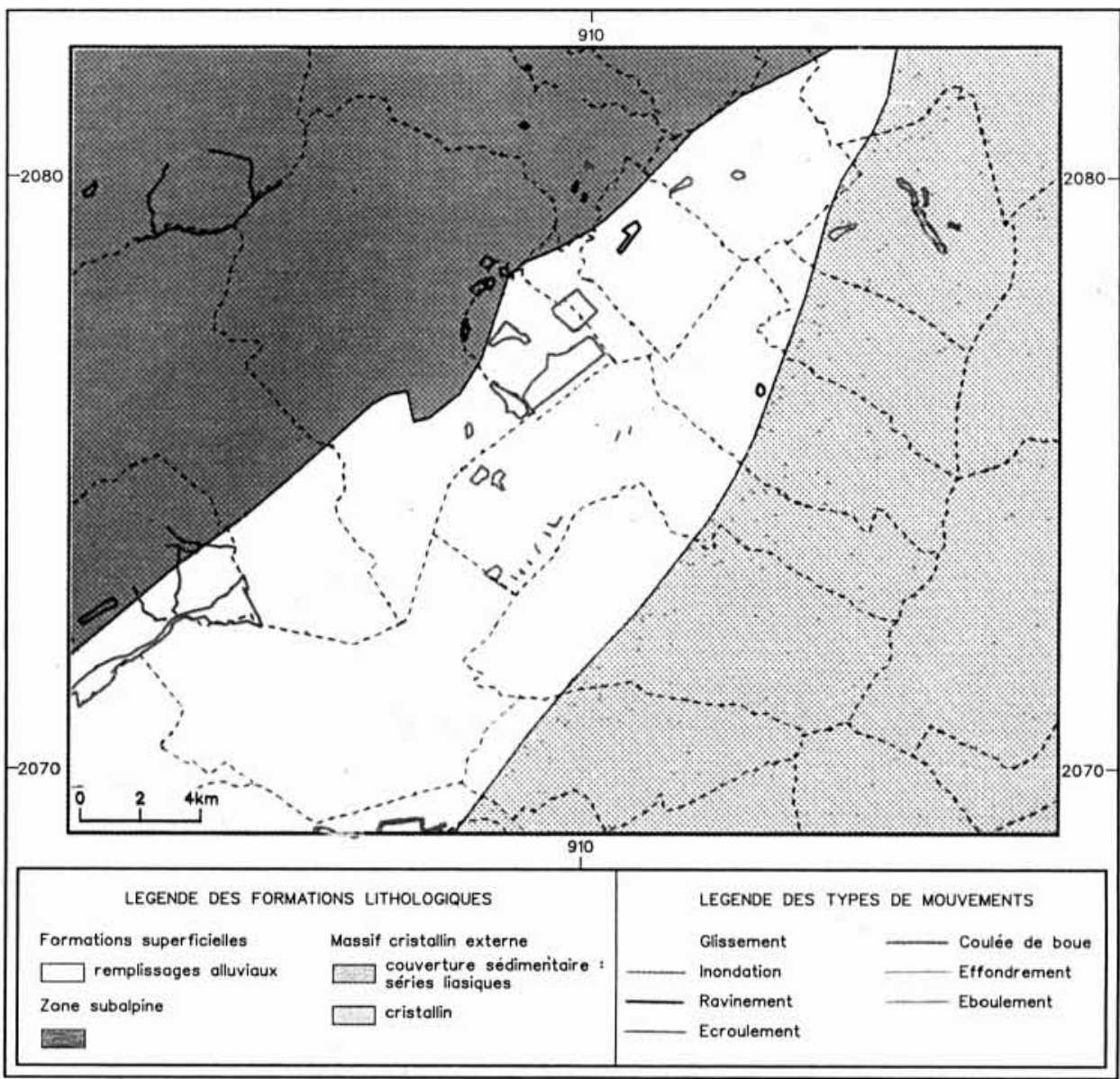

RAPPEL - En janvier et février 1990 après une longue période de sécheresse, alors que les stations alpines n'étaient que fort peu enneigées, plusieurs précipitations (pluie et neige) extrêmement abondantes se sont produites en quelques jours dans les Alpes du Nord, provoquant de nombreux phénomènes d'instabilité.

2.

C'est ce terme de dégradation des versants en montagne, abordé sur le plan de la géotechnique et des grands aménagements côté français, sur celui de la pédologie et des usages agricoles et forestiers côté italien, qui a été retenu pour fédérer les travaux des deux groupes. Au-delà des démarches évoquées dans le seul projet national, l'ambition, au niveau européen, est de montrer comment réaliser et mettre à disposition des systèmes d'information territoriaux permettant, grâce à l'expérience acquise par les divers acteurs en matière de prévention, de contrôler aussi efficacement que possible la dégradation des versants en montagne.
La proposition a été bien reçue à Bruxelles et soutenue par ces cofinancements européens.

L'échéance des deux projets est la même : ils doivent être terminés au printemps 1993. Géographiquement, les projets concerneront quatre sites pilote, deux en France (en Maurienne et dans le Trièves), et deux en Italie (en Valtellina et près de Florence en Toscane). Les systèmes d'information territoriaux seront établis à deux échelles différentes $\left(1 / 25000\right.$ pour une superficie de $600 \mathrm{~km}^{2}, 1 / 5000$ pour une superficie de $100 \mathrm{~km}^{2}$ ), sur la base d'informations saisies et traitées elles-mêmes à des échelles différentes (de la scène SPOT à la photographie aérienne rapprochée, de 
ESSA DE CORRELATION ENTRE LE CONTEXTE LITHOLOGIQUE REGIONAL ET LA TYPOLOGIE DES MOUVEMENTS DE TERRAN DANS LES ALPES DU NORD

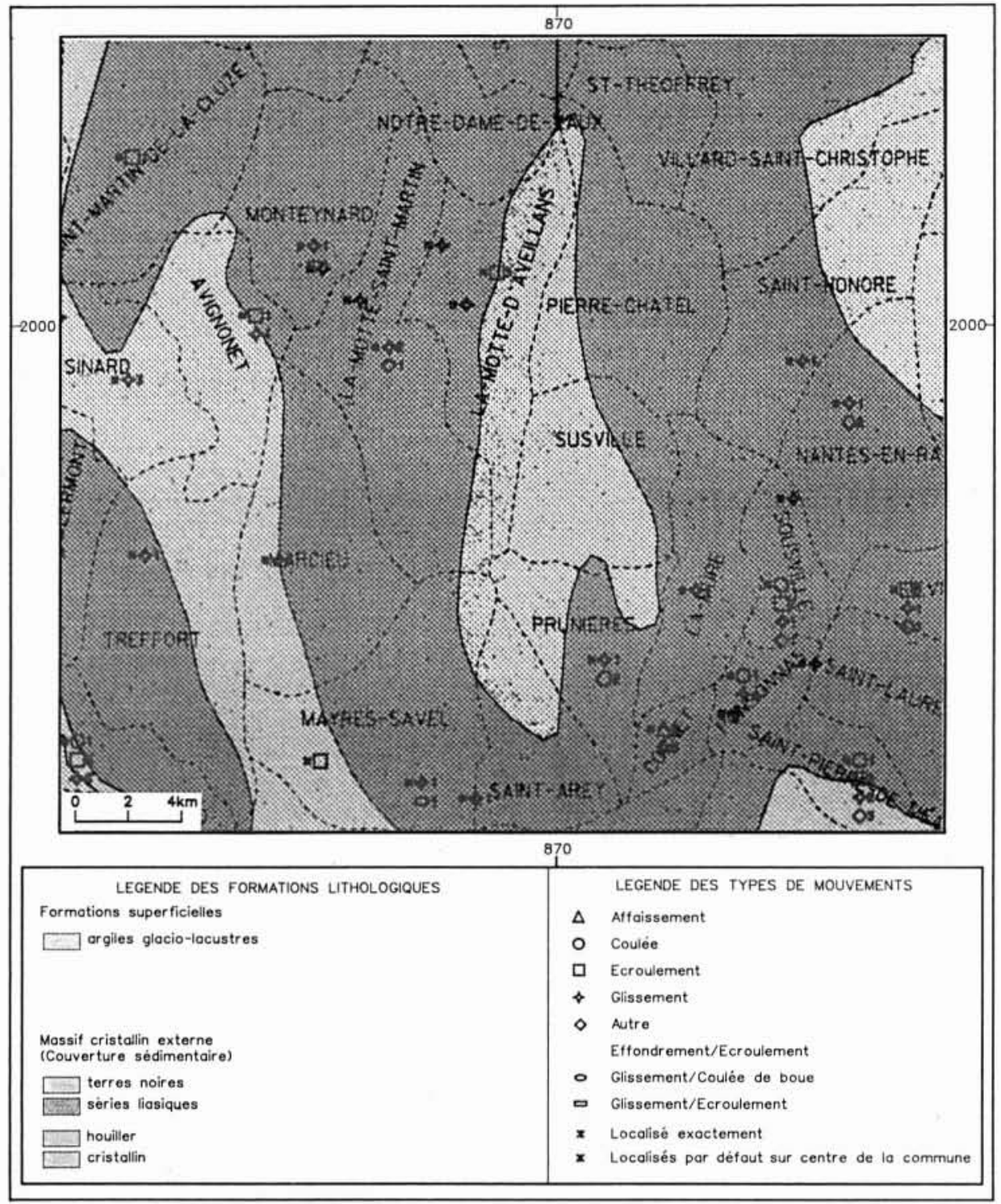

3.

la carte thématique régionale à l'interprétation locale de terrain). Ainsi entend-on montrer ce qu'il est licite de proposer comme méthodologie à chacune des échelles envisagées.

A terme, l'ensemble des recommandations issues des diverses composantes du projet devrait pouvoir être proposé comme un standard européen.

D'ores et déjà, le travail engagé a permis d'identifier et de formaliser deux projets de recherche à développer lors d'une prochaine étape et ayant un caractère transversal par rapport aux divers phénomènes naturels générateurs de risques en montagne:

- un programme de métrologie, surveillance et alerte des facteurs de risques,

- un programme d'« hydraulique distribuée " permettant de mieux comprendre le rôle de l'eau facteur de dégradation dans le cadre extrêmement complexe d'un bassin versant de montagne. 
LA CARTOGRAPHIE DES ZONES PRESUMEES INSTABLES
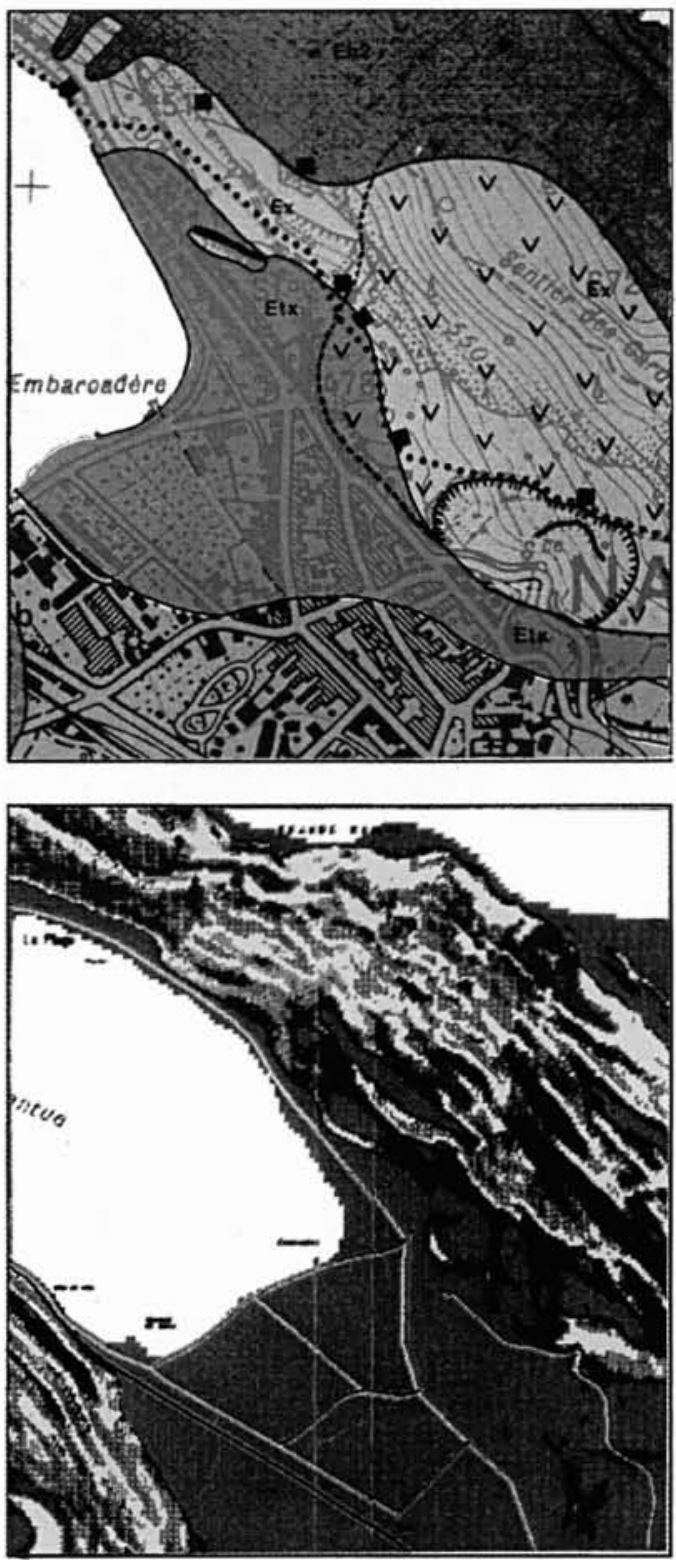

4b- VARIATION SPATIALE D'UN INDICE DE PREDISPOSITION AUX GLISSEMENTS DE TERRAN (obtenu par analyse combinatoire
multiparametres) 4a- LA CARTE ZERMOSS

(zones exposées à des mouvements du solet du sous-sol)

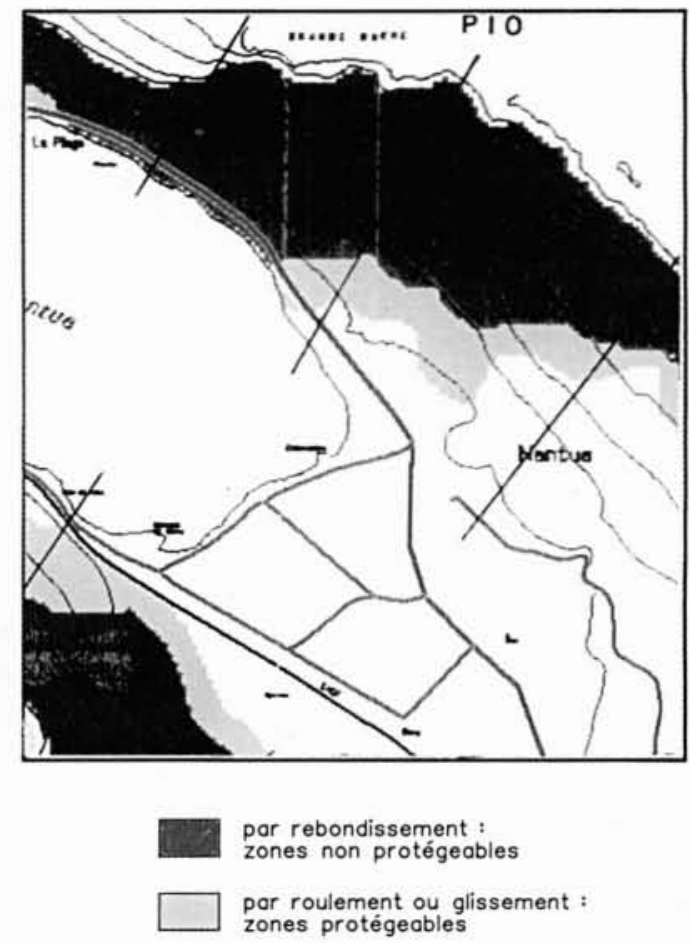

4c - ANALYSE DETERMINISTE DE L'EXTENSION DES ZONES EXPOSEES AUX RISQUES D'IMPACT DE BLOCS ROCHEUX

4.

\section{Références}

[1] Antoine P., Aste J.P., Azimı C., Desvarreux P., Giraud A., Rotheval J.P., 1989. Les mouvements de terrain dans les Alpes du Nord, Typologie des phénomènes. Méthodologie de la prévention. DATAR, Commissariat à l'Aménagement des Alpes du Nord.
[2] Aste J.P., Caille M., Gouisset Y., Viargues M., 1990. Le projet INVI. Développement du fichier informatique des mouvements de terrain. Application au département de l'Isère. Rapport BRGM R 30702 ENV 4S 90.

[3] Aste J.P., Chalivat P., Colleau A., 1989. Evaluation des différents phénomènes d'instabilité pouvant générer des risques sur la commune de Nantua (01). Rapport BRGM 89 SGN 430 RHA. 